\title{
A PERCEPÇÃO DE VALOR NA IMPLANTAÇÃO DE PMOs EM PEQUENAS E MÉDIAS EMPRESAS
}

\section{THE PERCEPTION OF VALUE IN THE IMPLEMENTATION OF PMOS IN SMALL AND MEDIUM-SIZED COMPANIES}

\author{
Vanessa Franco Pinheiro \\ Mestre em Administração pelo Centro Universitário Una \\ Centro Universitário Una - Una. \\ Belo Horizonte, Minas Gerais - Brasil. \\ vanessafrancopinheiro@yahoo.com.br \\ Danilo de Melo Costa \\ Doutor em Administração pela Universidade Federal de Minas Gerais (UFMG), \\ com período sanduíche na York University (Canadá). \\ Universidade Fumec - FUMEC. \\ Centro Universitário Una - Una. \\ Belo Horizonte, Minas Gerais - Brasil. \\ danilomct@gmail.com
}

Resumo: A gestão de projetos se apresenta como uma ferramenta importante para manter a competitividade empresarial e o diferencial pode estar na implementação de um Project Management Office (PMO) como uma alternativa para as organizações. Contudo, este assunto ainda é um paradigma nas organizações de pequeno e médio porte, que muitas vezes apresentam dificuldades e acabam negligenciando a criação de escritórios de gerenciamento de projetos. Diante deste contexto, este estudo tem como objetivo identificar, junto às lideranças executivas de empresas de pequeno e médio porte, quais os benefícios são esperados com a implantação de uma área de PMO. Com vista a atender ao objetivo proposto, foi realizada uma pesquisa exploratória com abordagem qualitativa e a coleta de dados a gestores de empresas filiadas ao PMI Minas Gerais. Os resultados apontam que os benefícios esperados estão relacionados principalmente a um melhor controle dos projetos, a confiabilidade e disponibilidade das informações, ao andamento dos projetos e a necessidade de um melhor balanceamento na alocação dos recursos humanos. Finalmente, conclui-se que estabelecer uma estratégia de implantação em pequenas e médias empresas de forma otimizada, com entregas parciais e que gere resultados a curto, médio e longo prazo pode ser o caminho para manter a continuidade de um PMO dentro da organização.

Palavras-chave: Gerenciamento de projetos. Escritório de Gerenciamento de Projetos EGP. Benefícios e serviços EGP.

Abstract: Project management presents itself as an important tool to maintain business competitiveness and the difference may be in the implementation of a Project Management Office (PMO) as an alternative for organizations. However, this issue is still a paradigm in small and medium-sized organizations, which often have difficulties and end up neglecting the creation of project management offices. Given this context, this study aims to identify, in a research with the executive leaders of small and medium-sized companies, which benefits are expected with the implementation of a PMO area. In order to meet the proposed objective, an exploratory research with a qualitative approach and data collection was conducted to managers of companies affiliated to PMI Minas Gerais. The results show that the expected benefits are mainly related to better project control, the reliability and availability of information, the progress of projects and the need for a better balance in the allocation of human resources. Finally, it is concluded that establishing an implantation strategy in small and medium companies in an optimized way, with partial deliveries and that generates results in the short, medium and long term can be the way to maintain the continuity of a PMO in the organization.

Keyword: Project management. EGP Project Management Office. Benefits and EGP services.

Cite como

American Psychological Association (APA)

Pinheiro, V. F., \& Costa, D. M. (2021, jan./jun.). A percepção de valor na implantação de PMOs em pequenas e médias empresas. Revista Inovação, Projetos e Tecnologias - IPTEC, São Paulo, 9(1), 124-142.

https://doi.org/10.5585/iptec.v9i1.19483. 


\section{Introdução}

Com o aumento da concorrência e o cenário global se mostrando mais competitivo, cresce a necessidade de as empresas se tornarem cada vez mais organizadas e ágeis, sendo assim obrigadas a buscar a inovação, o aprimoramento e a profissionalização na condução de seus projetos.

Neste contexto, a gestão de projetos se apresenta como uma ferramenta importante para manter a competitividade empresarial e aponta que o diferencial pode estar na implementação de um Escritório de Gerenciamento de Projetos (EGP) ou Project Management Office (PMO) como uma solução auxiliar no crescimento do negócio, respeitando a cultura e a maturidade em gestão de projetos existente dentro das organizações (PMI, 2017).

Segundo o Project Management Institute (PMI, 2017), um PMO é uma estrutura organizacional que padroniza os processos de governança relacionados a projetos e facilita o compartilhamento de recursos, metodologias, ferramentas e técnicas. O PMO pode trazer grande contribuição para o aumento da maturidade, padronização, gestão e controle, porém o sucesso de um PMO depende de sua correta implantação.

No entanto, a implantação de um PMO ainda é um desafio para as empresas. Seja por desconhecimento, por medo ou por representar alguma mudança de poder, a implantação de uma estrutura para gerenciamento de projetos atrai simpatizantes e adversários (Prado, 2008). Pinto (2015) corrobora com esta informação, ao demonstrar que apenas 22\% das empresas brasileiras reconhecem o valor do PMO em todos os níveis da organização, contra $42 \%$ que reconhecem parcialmente nos níveis estratégicos e 35\% que reconhecem parcialmente nos níveis operacionais. Se considerar que tais estudos tiveram como público alvo as grandes empresas, quando se delimita às Pequenas e Médias Empresas (PME), a adoção e a percepção de valor são ainda menores, fazendo com o PMO se torne quase que um paradigma neste contexto.

Isso ocorre, pois, organizações deste porte muitas vezes apresentam dificuldades e acabam negligenciando a criação de escritórios de gerenciamento de projetos. Diante deste contexto, este estudo tem como objetivo identificar, junto às lideranças executivas de empresas de pequeno e médio porte, quais os benefícios são esperados com a implantação de uma área de PMO.

Para tanto, é necessário que a implantação de um PMO seja planejada e possua o apoio da alta direção, assim como dos seus demais clientes, sendo de grande importância que os seus objetivos estejam alinhados com os objetivos estratégicos da organização. 


\section{Escritório de gerenciamento de projetos}

Segundo Vargas (2005), por mais que se tenha evoluído tecnicamente, é possível perceber um ambiente que evoluiu muito mais, ou seja, hoje se é mais capaz que no passado, porém, esse aumento de capacidade é cada vez menor se comparado com o aumento na dinâmica do ambiente, sendo necessário, portanto, desenvolver mecanismos que reduzam esta diferença entre homem e ambiente.

Neste contexto, a implantação de escritórios de PMO nas organizações pode ser vista como uma forte ferramenta para garantir o alinhamento entre os objetivos dos projetos e a estratégia das organizações, podendo inclusive reduzir esta diferença causada por esta dinâmica de evolução constante do ambiente.

O PMO é uma estrutura de gerenciamento que padroniza os processos de governança relacionados com o projeto e facilita o compartilhamento de recursos, metodologias, ferramenta e técnicas. O PMO é uma área dentro de uma organização à qual são atribuídas várias responsabilidades relacionadas ao gerenciamento centralizado e coordenado dos projetos sob seu domínio. As responsabilidades de um PMO podem variar desde fornecer funções de suporte ao gerenciamento de projetos até ser responsável pelo gerenciamento direto de um projeto (PMI, 2017).

Para Kendall e Rollins (2003), se destacam entre os objetivos dos escritórios de projetos nas organizações: a) possibilitar a condução dos projetos de forma alinhada com os interesses da alta direção; b) construir conhecimentos e habilidades para melhorar a capacidade e qualidade das entregas; c) coletar, analisar e disseminar informações sobre o desempenho dos projetos; d) detectar e aprimorar processos de gerenciamento de projetos ineficientes nos projetos da organização; e) valorizar o treinamento e desenvolvimento de habilidades em gerenciamento de projetos; f) implementar serviços de apoio para gerenciamento de projetos. Observa-se que existem muitas definições acerca dos conceitos e serviços tipicamente prestados por um PMO, porém Aubry, Hobs e Thuillier (2008) constataram que muitas organizações implementam escritórios de gerenciamento de projetos sem um direcionamento claro de qual o papel institucional do PMO em relação às necessidades organizacionais.

Hobbs, Aubry e Thuillier (2008) constataram através da realização de pesquisas uma grande variedade tanto na forma quanto nas funções dos PMOs. Além disso, na maioria dos casos, os PMOs são estruturas com grande instabilidade nas organizações. 
Diante deste contexto, observa-se a importância de um forte alinhamento institucional no processo de implantação de um PMO, sem o qual os riscos de insucesso neste processo podem caracterizar uma realidade cada vez mais frequente nas organizações.

\subsection{Implantação de PMOs: fatores motivacionais facilitadores e dificultadores}

A implementação de uma metodologia em gerenciamento de projetos, conjugada com a criação de um PMO, promove uma gestão de projetos mais estruturada, centralizada e alinhada com a nova estratégia da organização, proporcionando uma melhor visão e controle dos projetos empreendidos, que no passado eram carentes (Abe \& Carvalho, 2006).

Gold, Malhotra e Segars (2001) e Cepeda e Vera (2007) atribuem que os fatores que afetam diretamente o sucesso da gestão do conhecimento em projetos e consequentemente influenciam positivamente uma atuação de sucesso no PMO são as dimensões: Processos e Organização, Cultura e Liderança e Tecnológicos.

Existem diversos autores que corroboram com a tese de que implementar um PMO traz benefícios à organização, porém não se pode afirmar que de uma maneira geral essa posição seja uniforme. Na literatura observa-se que existem autores apontando razões tanto para implementação quanto para não implementação de um PMO.

O quadro 1 apresenta os motivadores e facilitadores encontrados com base em uma revisão de literatura, para criação de PMOs:

Quadro 1 - Motivos para implementar um PMO Continua

\begin{tabular}{|l|l|}
\hline \multicolumn{1}{|c|}{ Motivadores e Facilitadores } & \multicolumn{1}{c|}{ Autores } \\
\hline $\begin{array}{l}\text { Aperfeiçoar o gerenciamento de projetos e reduzir o } \\
\text { número de projetos problemáticos. }\end{array}$ & $\begin{array}{l}\text { Bernstein (2000) } \\
\text { Cleland e Ireland (2002) } \\
\text { Crawford (2002) } \\
\text { Dai e Wells (2004) } \\
\text { Desouza e Evaristo (2006) } \\
\text { Englund, Grahan e Dinsmore (2003) } \\
\text { Maximiliano e Anselmo (2006) } \\
\text { Rad (2001) }\end{array}$ \\
\hline $\begin{array}{l}\text { Usar os recursos de modo mais eficiente em ambiente } \\
\text { de múltiplos projetos. }\end{array}$ & $\begin{array}{l}\text { Cleland e Ireland (2002) } \\
\text { Dai e Wells (2004) } \\
\text { Patah (2004) }\end{array}$ \\
\hline $\begin{array}{l}\text { Melhorar a qualidade na implementação de projetos } \\
\text { estratégicos. }\end{array}$ & $\begin{array}{l}\text { Dai e Wells (2004) } \\
\text { Bernstein (2000) } \\
\text { Block e Frame (1998) } \\
\text { Patah (2004) }\end{array}$ \\
\hline $\begin{array}{l}\text { Facilitar a transferência de conhecimento sobre } \\
\text { gerenciamento de projetos na organização. }\end{array}$ & $\begin{array}{l}\text { Bernstein (2000) } \\
\text { Desouza e Evaristo (2006) }\end{array}$ \\
\hline
\end{tabular}




\begin{tabular}{|l|l|}
\hline \multicolumn{1}{|c|}{ Motivadores e Facilitadores } & Conclusão \\
\hline $\begin{array}{l}\text { Ter acesso às práticas de gerenciamento de projetos } \\
\text { consideradas como as melhores pelo mercado. }\end{array}$ & ITGI (2005) \\
\hline
\end{tabular}

Fonte: Adaptado de Spelta e Albertin (2009, p. 413-436).

Seguindo na direção contrária, ou seja, os fatores que dizem respeito aos dificultadores no processo de implementação de PMOs também foram identificados conforme Quadro 2:

Quadro 2 - Motivos para não implementar um PMO

\begin{tabular}{|l|l|}
\hline \multicolumn{1}{|c|}{ Motivadores e Dificultadores } & \multicolumn{1}{c|}{ Autores } \\
\hline $\begin{array}{l}\text { Não há provas de que melhora o desempenho dos } \\
\text { projetos. }\end{array}$ & $\begin{array}{l}\text { Dai e Wells (2004) } \\
\text { Englund, Grahan e Dinsmore (2003) } \\
\text { Jiang, Klein e Pick (2003) } \\
\text { Milosevic e Patanakul (2005) }\end{array}$ \\
\hline $\begin{array}{l}\text { Aumenta o custo fixo e pode não proporcionar } \\
\text { benefícios que justificam este aumento. }\end{array}$ & $\begin{array}{l}\text { Bernestein (2000) } \\
\text { Englund, Grahan e Dinsmore (2003) }\end{array}$ \\
\hline $\begin{array}{l}\text { Aumenta a burocracia. } \\
\text { Bernestein (2000) } \\
\text { Englund, Grahan e Dinsmore (2003) } \\
\text { Patah (2004) } \\
\text { Calderini e Cantamessa (1997) }\end{array}$ \\
\hline Cria conflitos entre setores e gerentes da organização. \\
$\begin{array}{l}\text { Valeriano (2001) } \\
\text { Englund, Grahan e Dinsmore (2003) } \\
\text { Patah (2004) }\end{array}$ \\
\hline $\begin{array}{l}\text { Perda de talentos para o gerenciamento de projetos. } \\
\text { implementar. }\end{array}$ & $\begin{array}{l}\text { Valeriano (2001) } \\
\text { Englund, Grahan e Dinsmore (2003) }\end{array}$ \\
\hline
\end{tabular}

Fonte: Adaptado de Spelta e Albertin (2009, p. 413-436).

Diante deste contexto é de fundamental importância que a implantação de um PMO leve em consideração os aspectos apresentados nas tabelas supracitadas para que seja possível minimizar os riscos de um inerente fracasso em virtude de uma falta de alinhamento mais profundo acerca dos reais objetivos que a organização pretende alcançar com a implantação de um PMO.

\subsection{Gestão de projetos em PMEs}

Para Almeida, Costa, Braga Junior e Porto (2018), as PMEs exercem um papel fundamental para a economia, contudo, são mais sensíveis aos abalos econômicos, justamente 
por sua limitação de atuação. Isso reforça a necessidade de profissionalizarem seus processos e gestão.

Geremia, Schmitt e Zanella (2020) também explicam que as pequenas e médias empresas muitas vezes carecem de uma maior profissionalização de sua gestão. Por este motivo, a gestão de projetos se torna um caminho seguro e responsável para trazer os resultados esperados para este tipo de organização.

A possibilidade de maior formalização dos processos, também é um fator de diferenciação para esta implantação, conforme explicam Vinagre et al. (2017). Para os autores, está metodologia e uma melhor organização dos processos servem de subsídio inclusive para implantarem inovações no mercado.

Massa, Figueiredo, Loiola e Passos (2020) corroboram com tal reflexão quando explicam que as PMEs são diferentes das grandes organizações e por isso precisam de uma abordagem específica. A adoção de uma gestão de projetos tradicional, sem a adaptação a este tipo e porte de organização, gerará gasto e tempos desnecessários, o que pode inviabilizar a iniciativa.

Por este motivo, estudar a percepção de valor de um PMO para este segmento se torna necessário, uma vez que são incipientes os estudos sobre a aplicação de gestão de projetos em PMEs (Sousa, Santos, Maccari \& Mazzieri, 2020; Kozlowski \& Matejun, 2016).

\section{Metodologia}

A classificação desta pesquisa, quanto à abordagem é qualitativa (Godoy, 1995). Foram realizadas entrevistas semiestruturadas junto a líderes executivos de empresas de pequeno e médio porte, filiados ao PMI-MG e previamente selecionados de forma intencional, com o objetivo de identificar quais benefícios esperados orientam os gestores no processo de implantação de um PMO em pequenas e médias empresas.

A pesquisa pode ser classificada, quanto aos meios, como sendo um estudo de caso (Yin, 2005; Gerhart \& Silveira, 2009) em pequenas e médias empresas filiadas ao PMI-MG que possuem um PMO implantado.

A amostra foi intencional o que para Stevenson e Farias (1981, p. 508), trata-se de “[...] uma amostragem não probabilística, subjetiva ou por julgamento, onde a variabilidade amostral não pode ser estabelecida com precisão". Contudo, foram selecionadas somente organizações de pequeno e médio porte filiadas ao PMI Minas Gerais Chapter (PMI-MG), como forma de 
evitar possíveis desvios e assegurar que são organizações que seguem as recomendações internacionais do PMI.

Para escolha da amostra foram encaminhados 2.791 e-mails para membros da instituição PMI-MG. Esta chamada alcançou 242 interessados que acessaram um formulário e preencheram um questionário com sete perguntas, que tinha por objetivo delimitar a amostra com base nas premissas da pesquisa. Após o atendimento das premissas este número reduziu para 36 empresas.

A partir de então, foram realizadas as entrevistas com lideranças executivas de empresas de pequeno e médio porte, filiados ao PMI-MG, de forma a compreender as percepções destes executivos, até que ocorresse o esgotamento do tema, sendo utilizado, portanto o princípio da saturação teórica (Glaser \& Strauss, 1967).

Ao longo do processo de entrevistas observou-se que a partir da sétima entrevista, o fenômeno da saturação teórica se apresentou sendo percebido um aumento da frequência dos termos: melhor controle e disponibilidade das informações, falta engajamento das equipes, estratégia desconhecida ou não disseminada, apoio à tomada de decisão, ausência de padrões e processos, faltam recursos humanos, falta uma comunicação mais integrada e baixa maturidade em gestão de projetos.

O instrumento de coleta de dados foi um questionário semiestruturado composto por 15 questões em formato de escala do tipo Likert de 7 pontos. Foi solicitado aos respondentes que manifestassem o nível de concordância com cada uma das 15 questões estabelecidas, além de responder verbalmente o motivo da referida escolha. Diante deste contexto e visando aprofundar o processo de análise dos dados, foram criadas quatro categorias que agruparam as 15 perguntas pertencentes ao questionário semiestruturado, conforme quadro 3. 
Quadro 3 - Agrupamento das perguntas em categorias

\begin{tabular}{|c|c|c|}
\hline Ordem & Perguntas & Categorias \\
\hline 1 & $\begin{array}{l}\text { Na minha organização, o PMO oferece melhor comunicação com o nível } \\
\text { executivo. }\end{array}$ & \multirow{3}{*}{$1^{\mathrm{a}}$ Comunicação } \\
\hline 2 & $\begin{array}{l}\text { Na minha organização, o PMO promove melhor comunicação entre a equipe } \\
\text { do projeto. }\end{array}$ & \\
\hline 3 & $\begin{array}{l}\text { Nosso PMO promove melhor comunicação e integração entre as diversas } \\
\text { áreas da organização. }\end{array}$ & \\
\hline 4 & $\begin{array}{l}\text { A adoção de um PMO possibilitou atingirmos estimativas/reduções dos } \\
\text { prazos e custos mais confiáveis. }\end{array}$ & \multirow{4}{*}{$2^{\mathrm{a}}$ Eficiência } \\
\hline 5 & $\begin{array}{l}\text { Após a adoção de um PMO percebemos uma melhoria na qualidade e um } \\
\text { maior compromisso com os resultados dos projetos. }\end{array}$ & \\
\hline 6 & $\begin{array}{l}\text { Após a adoção de um PMO, percebemos uma maior confiabilidade e } \\
\text { disponibilidade nas informações apresentadas. }\end{array}$ & \\
\hline 12 & $\begin{array}{l}\text { Após a adoção de um PMO, percebemos um aumento de produtividade dos } \\
\text { colaboradores. }\end{array}$ & \\
\hline 8 & $\begin{array}{l}\text { Na minha organização o PMO oferece maior visibilidade do andamento dos } \\
\text { projetos. }\end{array}$ & \multirow{4}{*}{$3^{a}$ Controle } \\
\hline 11 & $\begin{array}{l}\text { Após a adoção de um PMO, percebemos um melhor controle, de maneira } \\
\text { geral, sobre os projetos. }\end{array}$ & \\
\hline 13 & $\begin{array}{l}\text { Após a adoção de um PMO, percebemos uma maior clareza na definição de } \\
\text { papéis e responsabilidades. }\end{array}$ & \\
\hline 15 & $\begin{array}{l}\text { Na minha organização o PMO promove a alocação de recursos entre projetos } \\
\text { e proporciona uma maior visibilidade da demanda por recursos. }\end{array}$ & \\
\hline 14 & $\begin{array}{l}\text { Após a adoção de um PMO, percebemos uma maior satisfação dos clientes } \\
\text { dos projetos. }\end{array}$ & \multirow{4}{*}{$4^{\mathrm{a}}$ Estratégia } \\
\hline 7 & $\begin{array}{l}\text { A adoção de um PMO possibilitou maior previsibilidade e agilidade na } \\
\text { tomada de decisão. }\end{array}$ & \\
\hline 9 & $\begin{array}{l}\text { Após a adoção de um PMO, percebemos maior visibilidade da relação entre } \\
\text { projetos e estratégia. }\end{array}$ & \\
\hline 10 & $\begin{array}{l}\text { Após a adoção de um PMO, percebemos maior comprometimento do nível } \\
\text { executivo com os projetos. }\end{array}$ & \\
\hline
\end{tabular}

Fonte: Elaborado pelos autores.

A relação dos entrevistados com a respectiva nomeação que é utilizada para referenciálos nesta pesquisa, é apresentada no quadro 4. As entrevistas duraram em torno de 60 minutos: 
Quadro 4 - Agrupamento das perguntas em categorias

\begin{tabular}{|c|c|c|c|c|}
\hline Nomeação & Cargo & Segmento & $\begin{array}{c}\mathrm{N}^{\mathrm{o}} \mathrm{de} \\
\text { Empregados }\end{array}$ & Entrevista \\
\hline SQ & $\begin{array}{l}\text { Gestora de } \\
\text { Projetos }\end{array}$ & $\begin{array}{l}\text { Engenharia Consultiva } \\
\text { - Usinas Hidrelétricas }\end{array}$ & $\begin{array}{l}\text { Entre } 10 \text { e } 49 \\
\text { empregados }\end{array}$ & $\begin{array}{r}\text { Vídeo } \\
\text { conferência }\end{array}$ \\
\hline RV & $\begin{array}{l}\text { Diretor de } \\
\text { Projetos }\end{array}$ & $\begin{array}{l}\text { Geração de Energia de } \\
\text { Fontes Renováveis }\end{array}$ & $\begin{array}{r}\text { Entre } 100 \text { e } \\
499 \text { empregados }\end{array}$ & $\begin{array}{r}\text { Vídeo } \\
\text { conferência }\end{array}$ \\
\hline SF & $\begin{array}{l}\text { Diretor de } \\
\text { Operações }\end{array}$ & Engenharia Geotécnica & $\begin{array}{l}\text { Entre } 50 \text { e } 99 \\
\text { empregados }\end{array}$ & Presencial \\
\hline RG & $\begin{array}{l}\text { Coordenador de } \\
\text { PMO }\end{array}$ & Energia & $\begin{array}{l}\text { Entre } 10 \text { e } 49 \\
\text { empregados }\end{array}$ & $\begin{array}{r}\text { Vídeo } \\
\text { conferência }\end{array}$ \\
\hline MP & $\begin{array}{l}\text { Analista de } \\
\text { Projetos }\end{array}$ & $\begin{array}{c}\text { Consultoria em } \\
\text { Gerenciamento de Projetos }\end{array}$ & $\begin{array}{l}\text { Entre } 10 \text { e } 49 \\
\text { empregados }\end{array}$ & $\begin{array}{r}\text { Vídeo } \\
\text { conferência }\end{array}$ \\
\hline DL & $\begin{array}{l}\text { Engenheiro de } \\
\text { Planejamento }\end{array}$ & $\begin{array}{l}\text { Planejamento, } \\
\text { Consultoria e Engenharia }\end{array}$ & $\begin{array}{l}\text { Entre } 50 \text { e } 99 \\
\text { empregados }\end{array}$ & $\begin{array}{r}\text { Vídeo } \\
\text { conferência }\end{array}$ \\
\hline MM & $\begin{array}{l}\text { Gerente de } \\
\text { Projetos }\end{array}$ & $\begin{array}{l}\text { Desenvolvimento } \\
\text { Humano e Social }\end{array}$ & $\begin{array}{l}\text { Entre } 10 \text { e } 49 \\
\text { empregados }\end{array}$ & $\begin{array}{r}\text { Vídeo } \\
\text { conferência }\end{array}$ \\
\hline
\end{tabular}

Fonte: Elaborado pelos autores.

No que tange ao perfil dos entrevistados, quanto ao tempo na empresa, $43 \%$ dos entrevistados estão na empresa entre 6 a 10 anos, 29\% estão na empresa entre 1 a 5 anos e 14\% estão na empresa acima de 20 anos. No tocante ao grau de escolaridade, $71 \%$ dos entrevistados possuem especialização e $29 \%$ possuem mestrado. Já em relação aos projetos desenvolvidos (clientes externos ou internos), $71 \%$ das empresas desenvolvem projetos para clientes externos.

Em relação a parte específica da estrutura de PMO, quanto ao tempo de existência do PMO, $72 \%$ das empresas tem menos de 2 anos de existência. Constatou-se também que $71 \%$ possuem PMOs com 2 a 3 profissionais. Já, em relação a duração média dos projetos, $57 \%$ dos projetos são de duração inferior a 9 meses, sendo que deste total $43 \%$ são inferiores a 6 meses. Por fim, no tocante a maturidade em gestão de projetos, $57 \%$ considera o nível médio, contra $14 \%$ pouco baixa e $29 \%$ muito baixa o que evidencia que as empresas de pequeno e médio porte ainda precisam avançar muito nos seus processos de gestão de projetos.

Após a realização das entrevistas, os dados foram analisados e os benefícios esperados foram agrupados em quatro categorias: Comunicação, Eficiência, Controle e Estratégia, com o objetivo de buscar afinidades e ao mesmo tempo aprofundar os resultados, conforme apresentado a seguir.

$\mathrm{Na}$ etapa de análise e interpretação dos dados da pesquisa, as entrevistas semiestruturadas foram submetidas a técnica de análise de conteúdo categorial, visando identificar quais os critérios são comumente utilizados pelos líderes executivos no processo de 
decisão pelo investimento em estruturação de projetos e implantação de PMOs (Bardin, 1977; Gerhardt \& Silveira, 2009).

\section{Análise e discussão dos resultados}

Este capítulo tem por objetivo descrever e analisar os resultados obtidos com a pesquisa. Após a realização das entrevistas, os dados foram analisados e os benefícios esperados foram agrupados em quatro categorias: Comunicação, Eficiência, Controle e Estratégia, com o objetivo de buscar afinidades e ao mesmo tempo aprofundar os resultados, conforme apresentado a seguir.

\subsection{Categoria comunicação}

Ao se analisar a relação do PMO com a Comunicação em Nível Executivo, a maioria dos entrevistados reconhece de forma positiva e agregadora a comunicação do PMO com o nível executivo da organização. Porém alguns mencionam que há um certo distanciamento com a estratégia organizacional devido à falta de maturidade em gestão da organização, fazendo com que o PMO assuma um papel mais operacional e menos estratégico. O entrevistado DL destaca “[...] falta engajamento do executivo - o PMO acaba apoiando mais os Gerentes de Projetos". O entrevistado MP comenta “[...] estamos em processo de aprendizagem”.

No tocante a relação do PMO com a Comunicação junto a Equipe de Projetos, a maioria destaca que a implantação do PMO melhorou muito o nível de comunicação com a equipe técnica com destaque para o entrevistado SQ que menciona que "[...] a padronização estabelecida pelo PMO melhorou muito o processo de comunicação". Já o entrevistado RV reforça que “[...] o PMO conseguiu não só estabelecer uma melhor comunicação como também controlar este processo". O entrevistado SF também menciona "[...] a falta de maturidade em gestão de projetos é um fator dificultador no processo de comunicação com a equipe".

Por fim, sobre a relação do PMO com a Comunicação entre as Diversas Áreas da Organização, de uma maneira quase que geral os entrevistados concordam que a integração entre as áreas foi fortemente influenciada pelo PMO que estabeleceu padrões, disponibilizou informações e interagiu promovendo uma melhor comunicação entre as áreas da organização.

O entrevistado SF destaca que "[...] o PMO estabelece os padrões e disponibiliza as informações de forma integrada com diversas áreas da organização”. Já o entrevistado MP enfatiza “[...] a integração entre áreas é mais difícil devido a necessidade de interagir com diversas pessoas". 


\subsection{Categoria eficiência}

Ao se analisar a relação do PMO com a Redução dos Prazos e Custos, A maioria dos entrevistados apresenta uma realidade com dificuldades para medir a relação entre o PMO e a redução dos prazos juntamente com custos mais confiáveis. Mas entre os entrevistados há um consenso da importância e da necessidade de se evoluir nesta direção.

O entrevistado MP desabafa "[...] ainda estamos apagando incêndio" e o entrevistado DL comenta “[...] o controle não tem sido tão efetivo assim". Já o entrevistado MM reconhece que "[...] houve uma evolução, estamos no caminho certo" e o entrevistado RV foi enfático ao dizer "[...] não se pode afirmar que o PMO contribuiu nesta direção".

O processo de medição das variáveis prazo e custo em projetos é de alta complexidade e depende muito do segmento de negócios onde a empresa atua, bem como do grau de maturidade em gestão alcançado pela organização.

No que se refere a relação do PMO com a Melhoria na Qualidade e o Compromisso com os Resultados, todos os entrevistados apontam melhorias reais com a implantação do PMO evidenciando aumento de qualidade em diferentes processos dentro da organização. $\mathrm{O}$ entrevistado DL menciona que "[...] são mais pessoas cobrando prazos e acompanhando os projetos" e o entrevistado RV destaca "[...] a implantação do PMO foi uma mudança de conceito, de quebra de paradigma”. Teve ainda o entrevistado RG que comenta “[...] informações estruturadas levam, de fato, ao monitoramento e controle". Chama a atenção o entrevistado SQ que destaca “[...] os processos que não tem controle acabam destoando dos demais, deixando claro a necessidade de melhorias". Já o entrevistado SF menciona “[...] houve um ganho excessivo e transparente em relação a isso, tanto na qualidade quanto no compromisso de cumprir o que está sendo pedido".

Já sobre a relação do PMO com a Confiabilidade e Disponibilidade das Informações, de uma maneira geral os entrevistados reconhecem ganhos na disponibilidade das informações e na confiabilidade do que é apresentado. O entrevistado MP menciona "[...] fica mais fácil porque existe um padrão a ser seguido". O entrevistado RG destaca "[...] a centralização das informações em um conjunto de ferramentas usadas como fonte única fez com que todos pudessem acessar as informações que ficam disponíveis e são confiáveis”. O relato do entrevistado SQ chama atenção e menciona “[...] fazer o uso da informação estruturada para melhorar a qualidade na tomada de decisão é um ganho no processo de aprendizado organizacional, pois o uso de indicadores de desempenho evidencia a confiança dos executivos nas informações disponibilizadas". Há também o entrevistado MM que comenta “[...] 
reconheço dificuldades neste item em função da falta de recursos humanos para estruturar as informações e por isso considero que este processo está em evolução". Já o entrevistado SF comenta “[...] a disponibilidade das informações evoluiu e concordo 100\%, porém a confiabilidade ainda precisa avançar".

Finalmente, na relação do PMO com a Produtividade dos Colaboradores, os entrevistados destacaram que não conseguem ainda mensurar a produtividade dentro da empresa, mas a maioria observa que a implantação do PMO influenciou na produtividade, devido principalmente aos controles implementados que levaram as equipes a um maior comprometimento com os resultados e consequente impacto na produtividade.

O entrevistado MM comenta "[...] não tenho como mensurar isso hoje, a equipe apresenta dificuldade de engajamento e tem que ser acompanhada o tempo todo, dificultando ações nesta direção". O entrevistado RG comenta "[...] os processos devem ser simples para não impactar na produtividade da equipe que não está totalmente alocada em desenvolvimento de projetos, mas é percebido um aumento de produtividade, só não conseguimos mensurar”. O entrevistado SQ menciona “[...] uma vez que a empresa já está estruturada e possui os padrões estabelecidos, a equipe de especialistas gasta energia naquilo que é necessário, não precisando reinventar a roda".

\subsection{Categoria controle}

Ao se analisar a relação do PMO com o Andamentos dos Projetos, de uma maneira geral os entrevistados reconhecem que os PMOs têm atuado fortemente entregando informações que dão visibilidade do andamento dos projetos. A maioria das empresas possuem ferramentas de gestão de projetos e aqueles que não possuem fizeram um controle simples em planilhas. Muitos dos entrevistados destacaram basicamente o acompanhamento dos cronogramas (físico e financeiro) dos projetos e reportes periódicos para os executivos.

O entrevistado SF reconhece que “[...] antes do PMO havia uma ausência de informações e quando era necessário saber como estava o andamento de um projeto, somente perguntando diretamente ao coordenador. Hoje temos uma ferramenta de painel gerencial onde as principais informações estão lá”. Já o entrevistado MM destaca “[...] é possível ter uma melhor visibilidade do andamento dos projetos, mas sem ferramentas muito elaboradas, tudo simples e no Excel mesmo, com alguns faróis". O entrevistado RV menciona “[...] o controle atualmente ocorre através da ferramenta MS Project com reporte mensal de avanço físico". Já 
o entrevistado DL comenta “[...] a ferramenta não representa a realidade do andamento dos projetos, pois os cronogramas ficam constantemente desatualizados".

No tocante a relação do PMO com o Controle dos Projetos, de forma unânime todos os entrevistados concordaram que a implantação do PMO contribuiu muito para a melhoria dos controles existentes.

É importante destacar alguns relatos, como por exemplo o entrevistado MM que ressalta "[...] quem não mede não controla". O entrevistado RV destaca "[...] antes do PMO existiam controles, mas estavam longe de serem eficazes". Ainda o entrevistado SQ destaca “[...] hoje é possível enxergar onde está dando certo e onde está dando errado”. Já o entrevistado SF menciona "[...] hoje temos um controle maior e conseguimos direcionar melhor as nossas decisões".

Quando se analisa a relação do PMO com Papéis e Responsabilidades, a maior parte dos entrevistados reconhece que a implantação do PMO contribuiu para deixar mais claro os papéis e responsabilidades. O entrevistado DL relata "[...] a criação do Termo de Abertura do Projeto foi o primeiro passo e agora seria necessário evoluir para a definição da Matriz de Responsabilidades”. O entrevistado MM menciona “[...] após a explicação do conceito da Matriz RACI (Responsible, Accountable, Consulted, Informed), a equipe entendeu o que precisava ser feito para alcançar os objetivos do projeto lá na ponta”. Ainda teve o entrevistado SF que destacou "[...] após a definição dos papéis e responsabilidades as tarefas foram melhor direcionadas, faltando agora divulgar melhor e cobrar da equipe".

Finalmente, na relação do PMO com Alocação de Recursos, a grande maioria dos entrevistados reconhece a responsabilidade do PMO pelo processo de controle das alocações de recursos humanos. Merece destaque o entrevistado DL que menciona “[...] ocorrem conflitos de interesse no processo de alocação compartilhada, quando um recurso é demandado para diferentes projetos, e o PMO se apresenta com um olhar mais corporativo". O entrevistado RV também explica que "[...] funciona satisfatoriamente, porque o PMO consegue garantir a integração de recursos, de forma que não haja nem sobrecarga, nem gente ociosa".

\subsection{Categoria estratégia}

Ao se analisar a relação do PMO com uma maior Previsibilidade e Agilidade na Tomada de Decisão, de uma maneira geral os entrevistados apontam que as informações estão disponíveis, porém de forma desestruturada o que acaba dificultando o processo de tomada de decisão. O entrevistado DL menciona “[...] no nível operacional/ tático é perceptível, porém no 
nível estratégico nem tanto". O entrevistado SF destaca "[...] tem previsibilidade maior, mas a agilidade na tomada de decisão precisa estruturar melhor, mas o problema não é o PMO, mas sim as lideranças executivas e o mercado".

Já no que se refere a relação do PMO com a Estratégia, observa-se que a relação entre os projetos e a estratégia de negócios da empresa é baixa, porém os relatos remetem a uma necessidade real de ampliar e evoluir esta relação.

O entrevistado DL menciona “[...] há dificuldades em captar a estratégia da empresa”. O entrevistado RG ressalta “[...] não há um casamento entre a estratégia e o PMO, tendo muito espaço para avançar". O entrevistado SQ destaca “[...] existe uma relação direta porque o PMO atua fortemente com o nível executivo, porém não se pode dizer o mesmo em relação ao restante da organização, porém neste caso não se trata de PMO, mas sim da cultura organizacional que possui baixa maturidade em gestão".

Sobre a relação do PMO com o Nível Executivo, na maioria das vezes a implantação de um PMO ocorre por decisão do nível executivo das organizações. Só este fato, de forma isolada, justificaria o total interesse de que esta área contribua para o crescimento organizacional. $\mathrm{O}$ fato é que existem muitas interpretações quando o tema envolve comprometimento dos executivos. Sob este aspecto o PMO deve ter a capacidade de se adaptar conforme a cultura da organização e dos seus executivos, sendo alguns muito participativos/ atuantes e outros nem tanto.

Por fim, no tocante a relação do PMO com a Satisfação dos Clientes, a grande maioria concorda que a implantação do PMO gerou uma maior satisfação por parte dos clientes. O entrevistado RV afirma "[...] ficou mais organizado, houve um aumento na qualidade e o cliente diminuiu as reclamações". O entrevistado RG menciona “[...] o retorno positivo foi percebido mais no nível estratégico do que no operacional". Ainda teve o relato do entrevistado SF que destaca “[...] a empresa tem um controle maior e as informações hoje chegam mais fáceis, mas o aumento da satisfação não se deve exclusivamente ao PMO, mas também a qualidade técnica dos produtos". O entrevistado DL menciona “[...] existe um indicador que mede a satisfação do cliente, porém os números não refletem a realidade por razões, às vezes, políticas". Ainda teve o entrevistado SQ que comenta “[...] não foi percebida esta relação entre o PMO e uma melhoria na satisfação dos clientes".

\section{Conclusões}

Os resultados alcançados nesta pesquisa permitiram compreender não somente quais são os benefícios esperados pelas lideranças executivas na implantação de um PMO em 
empresas de pequeno e médio porte, mas também quais são as lacunas que ainda precisam ser exploradas por estas empresas.

Ao analisar as respostas para os 15 benefícios apontados, identificou-se que os principais benefícios esperados com a criação de uma área de PMO, em empresas de pequeno e médio porte, estão relacionados ao controle, a confiabilidade e disponibilidade das informações, ao andamento dos projetos e a alocação dos recursos humanos.

As lacunas aparecem quando os entrevistados apontam alguns benefícios esperados, porém pouco atendidos pelas estruturas atuais do PMO destas empresas, a saber: reduções no prazo e no custo dos projetos, baixo comprometimento do nível executivo, pouca visibilidade entre projetos e estratégia e dificuldades em relação a produtividade da equipe.

É importante destacar que não há um caminho único a ser perseguido, porque a cultura organizacional leva a direções distintas, porém quando se consegue mapear os benefícios e os serviços tipicamente prestados por PMOs, em empresas de pequeno e médio porte, o processo de implantação pode ser melhor direcionado.

Soma-se a isso a importância de se conhecer às expectativas das lideranças executivas. É fundamental que haja uma clareza e um forte direcionamento no processo de implantação de um PMO, o que facilitará, em muito, o alcance dos resultados esperados.

Ainda assim e mesmo considerando que há uma clareza dos benefícios esperados, recomenda-se que sejam priorizados os processos críticos, porém sem deixar de lado que a implantação de alguns destes processos pode ser de alta complexidade e possivelmente de difícil implantação, podendo deixar o PMO exposto e sendo questionado pela demora nos resultados.

Diante disso e considerando a dinâmica do mercado cada vez mais ágil, esta demora pode fazer com que o PMO perca a credibilidade perante a liderança executiva e a organização. Portanto recomenda-se que seja definida uma priorização que leve em consideração não somente os processos críticos, mas também o grau de dificuldade para implantação dos mesmos

Como contribuições práticas, se destaca a oportunidade das organizações de Pequeno e Médio Porte (PMEs) compreender não só os benefícios esperados como evidenciar, a partir destes casos, as principais vantagens de se estruturar um PMO. Já, como contribuição teórica, o estudo avança a partir de uma lacuna onde os principais estudos sobre adoção de PMO se concentram somente em grandes empresas. Com isso espera-se reduzir o distanciamento atual entre as empresas de grande porte em relação às pequenas e médias, desmistificando possíveis fatores que possam estar dificultando a proliferação de PMOs neste segmento de mercado. 
Sugere-se para a realização de estudos futuros, a ampliação da amostra, saindo do universo das empresas filiadas somente ao PMI-MG e estendendo a pesquisa para outros estados brasileiros, porém mantendo a premissa de que o entrevistado é um filiado do PMI e as melhores práticas, preconizadas por esta instituição, são conhecidas e possivelmente utilizadas dentro destas organizações.

Destaca-se também que neste estudo foi realizada uma pesquisa qualitativa, onde o modelo criado poderia ser testado também sob uma perspectiva quantitativa, para futuras análises.

Por fim, este trabalho evidenciou as carências e lacunas quando o tema é a adoção de PMO em pequenas e médias empresas, deixando assim o registro e o desejo de que futuras explorações possam ser realizadas na direção de contribuir e aprimorar cada vez mais esta comunidade.

Estabelecer uma estratégia de implantação de forma otimizada, com entregas parciais e que gere resultados a curto, médio e longo prazo pode ser o segredo para manter viva a semente do PMO dentro da organização.

\section{Referências}

Abre, C. K., \& Carvalho, M. (2006). Fatores críticos para implementação do Escritório de Projetos: um estudo de caso. Revista Gestão da Produção, Operações e Sistemas, 1(1), 61-74.

Almeida, J.M.S., Costa, P.R., Braga Junior, S.S., \& Porto, G.S. (2018). Capacidade relacional e desenvolvimento de novos produtos em pequenas empresas de base tecnológica. Revista de Empreendedorismo e Gestão de Pequenas Empresas, 7(3), 141-166. http://dx.doi.org/10.14211/regepe.v7i3.857

Aubry, M., Hobbs, B., \& Thuillier, D. (2008). Organisational project management: an historical approach to the study of PMOs. International Journal of Project Management, 26(1), 38-43. https://doi.org/10.1016/j.ijproman.2007.08.009

Bardin, L. (1977). Análise de conteúdo. Lisboa: Edições 70.

Bernstein, S. (2000). Project Offices in Practice. Project Management Journal, 31(4), 4-6.

Block, T., \& Frame, J. (1998). The Project Office. Menlo Park: Crisp Learning.

Calderini, M., \& Cantamessa, M. (1997). Innovation paths in product development: an empirical research. International Journal of Production Economics, 51 (1-2), 1-17. https://doi.org/10.1016/S0925-5273(97)00076-5. 
Cepeda, G., \& Vera, D. (2007). Dynamic capabilities and operational capabilities: A knowledge management perspective, Journal of Business Research, 60 (5), 426-437.

https://doi.org/10.1016/j.jbusres.2007.01.013

Cleland, D., \& Ireland, L. (2002). Gerência de Projetos. Rio de Janeiro: Reichmann \& Affonso Editores.

Crawford, L. (2002). The strategic project office: a guide to improving organizational performance. New York: Marcel Dekker.

Dai, C. X., \& Wells, W. G. (2004). An exploration of project management office features and their relationship to project performance. International Journal of Project Management, 22(7), 523-532. https://doi.org/10.1016/j.ijproman.2004.04.001

Desouza, K. C., \& Evaristo, J. R. (2006). Project Management Offices: a case of knowledgebased archetypes. International Journal of Information Management, 26(5), 414-423. https://doi.org/10.1016/j.ijinfomgt.2006.07.002

Englund, R. L., Grahan, R. J., \& Dinsmore, P. C. (2003). Creating the project office: a manager's guide to leading organizational change. San Francisco: Jossey-Brass.

Gehardt, T. E., \& Silveira, T. E. (2009). Métodos de pesquisa. Porto Alegre: Editora da UFRGS.

Geremia, C., Schmitt, D., \& Zanella, C. (2020). Nível de maturidade em gerenciamento de projetos: estudo de caso em empresa familiar de médio porte do ramo alimentício. Revista de Gestão e Projetos, 11(2), 110-132. https://doi.org/10.5585/gep.v11i2.16911

Glaser, B., \& Strauss, A. (1967). The discovery of grounded theory: strategies for qualitative research. New York: Aldine de Gruyter.

Godoy, A. S. (1995). Introdução à pesquisa qualitativa e suas possibilidades. Revista de administração de empresas, 35(2), 57-63. https://doi.org/10.1590/S003475901995000200008

Gold, A. H., Malhotra, A., \& Segars, A. H (2001). Knowledge management: an organizational capabilities perspective. Journal of Management Information Systems, 18(1), 185-214. https://doi.org/10.1080/07421222.2001.11045669

Hobbs, B., Aubry, M., \& Thuillier, D. (2008). The project management office as an organisational innovation. International Journal of Project Management, 26(5), 547-555. https://doi.org/10.1016/j.ijproman.2008.05.008

IT Governance Institute. (2005). Cobit 4.0: control objectives, management guidelines, maturit models. Rolling Meadows: ISACA.

Jiang, J. J., Klein, G., \& Pick, R. A. (2003). The impact of IS department organizational environments upon project team performances. Information \& Management, 40(3), 213-220. https://doi.org/10.1016/ S0378-7206(02)00005-8 
Kendall, G., \& Rollins, S. (2003). Advanced project portfolio management and the PMO Multiplying ROI at Warp Speed. Rio de Janeiro: J. Ross Publishing.

Kozlowski, R., \& Matejun, M. (2016). Characteristic features of project management in small and medium-sized enterprises. E+M Ekonomie a Management, 19(1), 33-48.

http://dx.doi.org/10.15240/tul/001/2016-1-003

Massa, N., Figueiredo, P. S., Loiola, E., \& Passos, F. U. (2020). Melhores práticas em gestão de projetos de novos produtos aplicadas a uma empresa da indústria da moda. Revista Gestão \& Planejamento, 21(1), 512-530. http://dx.doi.org/10.21714/2178-8030gep.v.21.5441

Maximiano, A. C. A., \& Ancelmo, J. L. (2006). Escritório de gerenciamento de projetos: um estudo de caso. Revista de Administração da USP, 41(4), 394-403.

https://doi.org/10.1590/S0080-21072006000400004

Milosevic, D., \& Patanakul, P. (2005). Standardized project management may increase development projects success. International Journal of Project Management, 23(3), 181-192. https://doi.org/10.1590/S0080-21072006000400004

Patah, L. A. (2004). Alinhamento estratégico de estrutura organizacional de projetos: uma análise de múltiplos casos. Dissertação de Mestrado, Universidade de São Paulo, São Paulo, Brasil.

Pinto, A. (2015). Pesquisa PMO Brasil 2015. Recuperado em 8 setembro, 2020, de https://pt.slideshare.net/AmericoPinto2/pesquisa-pmo-project-management-office-brasilbrasil-2015.

Project Management Institute [PMI] (2017). PMBOK - Guia do Conhecimento em Gerenciamento de Projetos (6. ed.). Campus Boulevar: Newtown Square.

Prado, D. (2008). Maturidade em Gerenciamento de Projetos. Nova Lima: INDG Tecnologia e Serviços Ltda.

Rad, P. F. (2001). Is your organization a candidate for Project Management Office (PMO)? AACE International Transactions, 7(1), 1-4.

Spelta, A., \& Albertin, A. (2009). O contexto da criação de escritórios de projetos nas áreas de TI: dois estudos de caso. Journal of Information Systems and Technology Management, 6(3), 413-436. https://doi.org/10.4301/S1807-17752009000300003

Stevenson, W. J., \& De Farias, A. A. (1981). Estatística aplicada à administração. São Paulo: Harbra.

Souza, R., Santos, T., Maccari, E., \& Mazieri, M. (2020). Proposição de um modelo de gerenciamento de projetos para Pequenas e Médias Empresas (PME). Revista de Empreendedorismo e Gestão de Pequenas Empresas, 9(3), 384-407. http://dx.doi.org/10.14211/regepe.v9i3.1475

Valeriano, L. (2001). Gerenciamento estratégico e administração por projetos. São Paulo: Makron Books. 
Vargas, R. (2005). Gerenciamento de projetos: estabelecendo diferenciais competitivos (6. ed.). Rio de Janeiro: Brasport.

Vinagre, L.M., Silva, S.W., Portugal, P.S., Souza, R.S., Gonçalves, J.E. \& Pereira, W.F. (2017). O processo sucessório em uma empresa familiar: um estudo de caso em um supermercado de Varginha-MG. Revista da Universidade Vale do Rio Verde, 15(1), 689-700. http://dx.doi.org/10.5892/ruvrd.v15i1.3401

Yin, R. K. (2005). Estudo de caso: planejamento e métodos (3. ed.). Porto Alegre: Bookman. 